02

\title{
Квантовый размерный эффект в сверхпроводящих пленках алюминия
}

\author{
(C) К.Ю. Арутюнов ${ }^{1,2}$, Е.А. Седов ${ }^{1}$, И.А. Голоколенов ${ }^{1,2}$, В.В. Завьялов ${ }^{1,2}$, Г. Константинидис ${ }^{3}$, \\ А. Ставринидис ${ }^{3}$, Г. Ставринидис ${ }^{3}$, И. Василиадис ${ }^{4}$, Т. Кехагиас ${ }^{4}$, Г.П. Димитракопулос ${ }^{4}$, \\ Ф. Комнину 4 , М.Д. Кроитору ${ }^{5,6}$, А.А. Шаненко
}

${ }^{1}$ Национальный исследовательский университет „Высшая школа экономики“, Москва, Россия

${ }^{2}$ Институт фризических проблем им. П.Л. Капицы РАН,

Москва, Россия

${ }^{3}$ Институт электронных структур и лазеров, Фонд исследований и технологии Греции (FORTH), Ираклион, Греция

${ }^{4}$ Департамент физики, Университет Аристотеля Салоники,

Салоники, Греция

${ }^{5}$ Институт теоретической фризики III, Университет Баройта,

Баройт, Германия

${ }^{6}$ Департамент физики, Центр точных и естественных наук (CCEN), Федеральный университет Пернамбуко, Пернамбуко, Бразилия

E-mail: karutyunov@hse.ru

Поступила в Редакцию 15 апреля 2019 г.

В окончательной редакции 22 апреля 2019 г.

Принята к публикации 24 апреля 2019 г.

Экспериментально исследовались высококачественные пленки алюминия на подложках из GaAs. Был обнаружен заметный рост критической температуры сверхпроводящего перехода с уменьшением толщины пленки. Эффект интерпретируется как проявление квантового размерного эффекта, влияющего как на плотность состояний электронов, так и на электрон-фононное взаимодействие.

Ключевые слова: сверхпроводимость, критическая температрура, тонкие пленки, квантовый размерный эффект.

DOI: $10.21883 /$ FTT.2019.09.48098.28N

\section{1. Введение}

Уже в самых ранних работах по сверхпроводимости было обнаружено, что критическая температура сверхпроводящего перехода $T_{c}$ тонких пленок может значительно отличаться от соответствующей величины для массивного образца [1]. Причем в ряде материалов с уменьшением толщины пленки наблюдается уменьшение $T_{c}$ (например, в ниобии [2]), а в других увеличение (например, в алюминии $[3,4]$ ). Несмотря на достаточно обширный экспериментальный материал и изобилие теоретических моделей, до настоящего времени в научном сообществе нет единого взгляда на природу этого явления. Одним из „объяснений“ является утверждение В.Л. Гинзбурга [5] о возможности формирования в реальных тонкопленочных образцах сложной системы слабосвязанных зерен, которая может привести к существенному отклонению $T_{c}$ от значения, ожидаемого для монокристаллической квазидвумерной системы идентичной толщины. Целью настоящей работы было экспериментальное исследование температуры сверхпроводящего перехода в тонких высококачественных пленках алюминия.

\section{2. Теория}

Вклад эффекта размерного квантования на сверхпроводящие свойства может быть понят на качественном уровне очень просто. Рассмотрим простой металл с параболическим законом дисперсии $E(\mathbf{p})$ (рис. 1, пунктирная кривая). Если один из размеров системы (например, в направлении $Z$ ) настолько мал, что квантовым размерным эффектом (КРЭ) нельзя пренебречь, то для любого направления в плоскости пленки $\{X, Y\}$ зависимость $e\left(\mathbf{p}_{\{X, Y\}}\right)$ вырождается в систему размерноквантованных уровней $E_{n}$ (рис. 1 , сплошные кривые). Вхождение каждого такого уровня в энергетическое „окно“ $\mu \pm \hbar \omega_{D}$, где $\mu-$ химический потенциал, а $\omega_{D}$ - дебаевская частота, соответствует условию резонанса, приводящего к сингулярности плотности состояний и, соответственно, возрастанию критической температуры $T_{c}$.

Для количественного анализа вклада квантового размерного эффекта на величину сверхпроводящего параметра порядка $\Delta(r)$ может быть использован формализм уравнений Боголюбова-де Жена [6,7]:

$$
\left(\begin{array}{cc}
H_{e} & \Delta(r) \\
\Delta^{*}(r) & -H_{e}^{*}
\end{array}\right)\left(\begin{array}{l}
u_{n}(r) \\
v_{n}(r)
\end{array}\right)=E_{n}\left(\begin{array}{l}
u_{n}(r) \\
v_{n}(r)
\end{array}\right),
$$




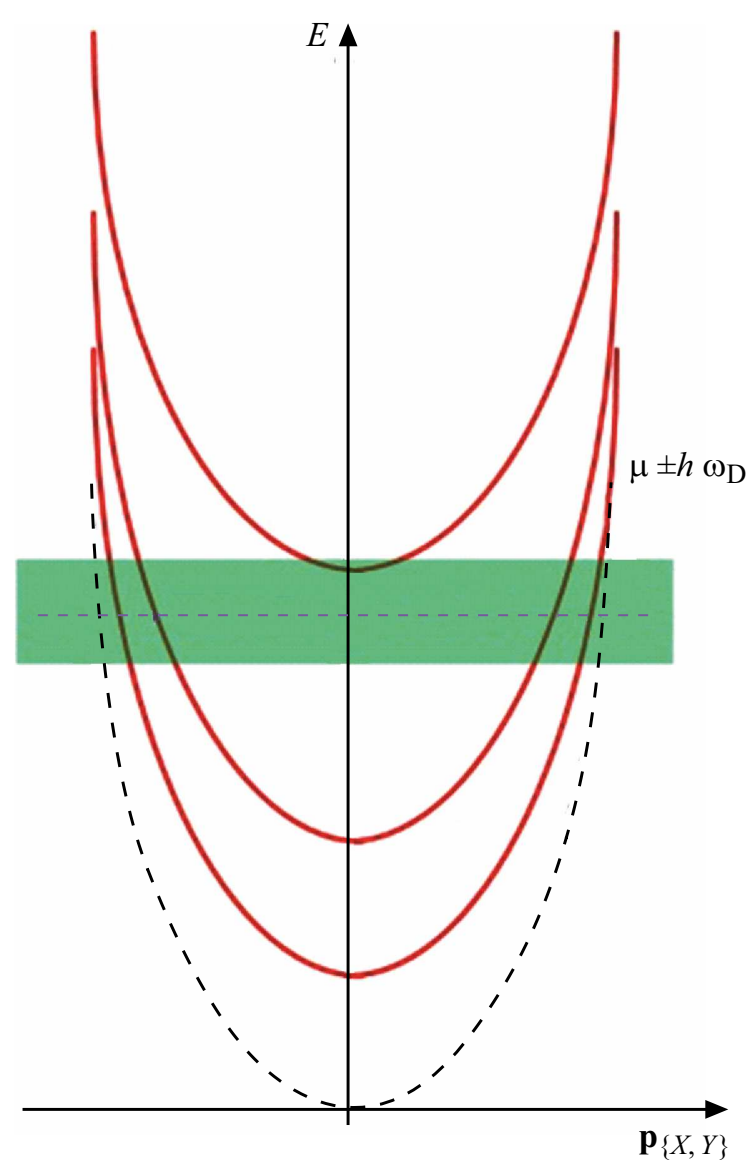

Рис. 1. Энергетический спектр металла с обычным параболическим законом дисперсии (пунктирная кривая) и в присутствии размерного квантования в направлении $Z$, перпендикулярном плоскости пленки $\{X, Y\}$ (сплошные линии). Вхождение каждого $n$-го размерно-квантованного уровня энергии $E_{n}$ в область $\mu \pm \hbar \omega_{D}$ соответствует условию резонанса, приводящего к сингулярности плотности состояний и, соответственно, возрастанию критической температуры $T_{c}$.

где одноэлектронный гамильтониан в нулевом магнитном поле выглядит как

$$
H_{e}=-\frac{\hbar^{2}}{2 m^{*}} \nabla^{2}+U(r)-\mu
$$

потенциал взаимодействия определяется собственными значениями $u_{n}(r)$ и $v_{n}(r)$, химическим потенциалом $\mu$ и константой электрон-фононного взаимодействия $g$ :

$$
U(r)=g \sum_{n}\left\{\left|u_{n}(r)\right|^{2} f_{n}+\left|v_{n}(r)\right|^{2}\left(1-f_{n}\right)\right\},
$$

где $f_{n}(E)$ - функция распределения Ферми-Дирака для $n$-го размерно-квантованного уровня энергии. Параметр порядка определяется как

$$
\Delta(r)=g \sum_{n}\left\{u_{n}(r) \nu_{n}^{*}(r)\left(1-2 f_{n}\right)\right\} .
$$

Суммирование производится по всем энергетическим уровням $E_{n}>0$ и с дополнительным условием $\left|\xi_{n}\right|<\hbar \omega_{D}$, где энергия отсчитывается от химического потенциала $\mu$ и одноэлектронная энергия $n$-го уровня дается выражением

$$
\xi_{n}=\int d^{3} r\left\{u_{n}^{*}(r) \hat{H}_{e} u_{n}(r)+v_{n}^{*}(r) \hat{H}_{e} v_{n}(r)\right\} .
$$

Плотность состояний $N(0)$ и химический потенциал $\mu$ находятся из условия нормировки, где $n_{e}-$ концентрация электронов:

$$
n_{e}=\frac{2}{V} \int d^{3} r \sum_{n}\left\{\left|u_{n}(r)\right|^{2} f_{n}+\left|v_{n}(r)\right|^{2}\left(1-f_{n}\right)\right\} .
$$

Для тонкой пленки с размерами $L_{z} \ll L_{x}, L_{y}$ граничные условия записываются как $\Delta(r)=\Delta(z)$ и $U(r)=U(z)$, что приводит к специфической форме собственных функций

$$
u_{l}(r)=\frac{e^{i k_{x} x}}{\sqrt{L_{x}}} \frac{e^{i k_{y} y}}{\sqrt{L_{y}}} \tilde{u}_{l}(z), \quad v_{l}(r)=\frac{e^{i k_{x} x}}{\sqrt{L_{x}}} \frac{e^{i k_{y} y}}{\sqrt{L_{y}}} \tilde{v}_{l}(z),
$$

где совокупность квантовых номеров $l=\left\{k_{x}, k_{y}, j\right\}$, квантовое число $j$ связано с квантованием спектра в направлении $Z$.

Учет граничных условий $\tilde{u}_{l}(0)=\tilde{u}_{l}\left(L_{z}\right)=0, \tilde{v}_{l}(0)=$ $=\tilde{v}_{l}\left(L_{z}\right)=0$ позволяет представить собственные функции в виде разложения по гармоникам

$$
\varphi_{l^{\prime}}=\sqrt{\frac{2}{L_{z}}} \sin \left(\frac{\pi\left(l^{\prime}+1\right) z}{L_{z}}\right), \quad l^{\prime}=0,1,2 \ldots
$$

Очевидно, что в тонких пленках электрон-фононное взаимодействие зависит от близости к границе. Было замечено [8], что отклонение константы электронфононного взаимодействия $g$ от своего значения $g_{b u l k}$ пропорционально $1 / M$, где $M-$ количество атомных монослоев в тонкопленочном образце, т.к. именно $1 / M$-я часть атомов находится у границы. В расчетах $[6,7]$ использовалось простое приближение для учета влияния конечной толщины пленки на константу электрон-фононного взаимодействия

$$
g=g_{0}-\frac{g_{1}\left(4 \pi a M / \lambda_{F}\right)}{M}=g_{0}-\frac{g_{1}\left(2 k_{F} a M\right)}{M},
$$

где $a-$ межатомное расстояние, $\lambda_{F}$ и $k_{F}-$ coответственно, фермиевская длина волны и волновой вектор, $\left[g_{0} N(0)\right]_{b u l k}=0.18-$ табулированная величина для массивного алюминия. Периодическая функция $g_{1}(0)=g_{1}(2 \pi)$ являлась подгоночным параметром.

Самосогласованное решение уравнений (1)-(9) позволяет рассчитать величину параметра порядка $\Delta(z)$. Критическая температура $T_{c}$ определяется как точка, при которой $\Delta(z)=0$ является единственным решением системы уравнений. Пример расчета зависимости критической температуры $T_{c}$ от толщины алюминие- 
вой пленки $d$, усредненной по ее флуктуациям, приведен на рис. 4.

\section{3. Образцы и методика эксперимента}

Методом электронно-лучевого испарения в вакууме $\sim 10^{-9} \operatorname{mbar}$ и молекулярно-лучевой эпитаксии были изготовлены пленки алюминия на подложках из GaAs. Толщина образцов варьировалась от 5 до $100 \mathrm{~nm}$, ширина $-500 \mu \mathrm{m}$, длина $-10 \mathrm{~mm}$. Качество пленок контролировалось методом просвечивающей электронной микроскопии высокого разрешения. Было установлено, что все изготовленные образцы являются поликристаллами, причем характерный размер кристаллитов в плоскости пленки сравним или превосходит толщину пленки (рис. 2). На границах зерен наблюдаются дефекты несоответствия кристаллических ориентаций. Однако никаких признаков присутствия чужеродных примесей обнаружено не было. Микроскопический анализ пленок,
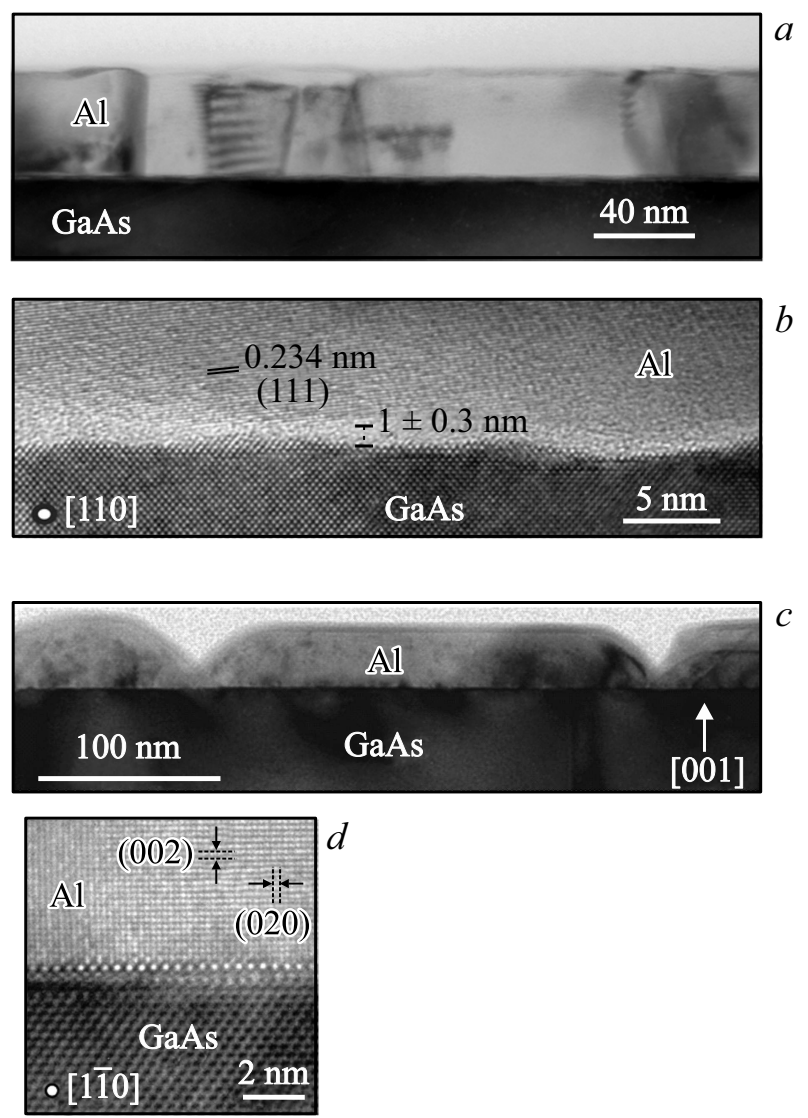

Рис. 2. Микрофотографии пленок алюминия на подложках из $\mathrm{GaAs}$, полученные методом просвечивающей электронной микроскопии высокого разрешения. $(a-b): 50 \mathrm{~nm}$ поликристаллическая пленка алюминия, полученная электроннолучевым испарением. $(c-d): 35 \mathrm{~nm}$ пленка, полученная методом молекулярно-лучевой эпитаксии. На фотографиях сверхвысокого разрешения $(b-d)$ хороша видна граница металл-подложка. полученных методом молекулярно-лучевой эпитаксии, показал, что размер кристаллитов в плоскости пленки - сушественно больше, чем для образцов, полученных электронно-лучевым испарением. Однако, желаемый технологический результат - квазидвумерный монокристалл - получить не удалось.

Экспериментальное исследование $R(T)$ зависимостей проводилось в четырехконтактной конфигурации как на постоянном, так и переменном токах. Измерительный ток (обычно от 1 до $10 \mu \mathrm{A}$ для измерений на постоянном токе, и от 0.1 до $1 \mu \mathrm{A}$ при фазочувствительных измерениях на частоте $17 \mathrm{~Hz}$ ) выбирался таким образом, чтобы его увеличение на порядок не приводило к заметному смещению температуры фазового перехода. В области температур выше $T=1.3 \mathrm{~K}$ измерения проводились в криостате прямой откачки ${ }^{4} \mathrm{He}$. Для работы в более низком температурном диапазоне использовался сорбционный ${ }^{3} \mathrm{He}$ криостат. Термометры в обеих системах были прокалиброваны по давлению паров ${ }^{4} \mathrm{He}$ и по реперным точкам сверхпроводящих переходов чистых массивных сверхпроводников (Ti, Al, Sn, In). Результирующая абсолютная погрешность определения температуры составляла $\sim 10 \mathrm{mK}$, в то время как относительная - менее $1 \mathrm{mK}$. Повторные измерения $T_{c}$ одного и того же образца в двух различных установках совпадали с точностью до нескольких $\mathrm{mK}$. Забегая вперед, можно отметить, погрешность измерения температуры была существенно меньше размерно-зависимого изменения $T_{c}$.

\section{4. Результаты и обсуждение}

Типичная зависимость сопротивления алюминиевых пленок от температуры $R(T)$ приведена на рис. 3 . Определяя критическую температуру сверхпроводящего перехода как точку, при которой сопротивление падает в 2 раза по отношению к нормальному состоянию, можно построить зависимость $T_{c}$ от толщины пленки $d$ (рис. 4). Четко прослеживается зависимость: чем тоньше пленка, тем выше критическая температура. Для самого тонкого образца с толщиной $5 \mathrm{~nm}$ критическая температуры увеличивается практически вдвое по сравнению со значением для массивного алюминия $T_{c}$ (массив $)=1.19 \mathrm{~K}$. Наши результаты хорошо согласуются с более ранними исследованиями $[3,4]$. Разумно предположить, что структура слабосвязанных звеньев [5], которая могла бы гипотетически сформироваться, должна была бы радикально отличаться для пленок различного качества, выращенных на различных подложках. Следовательно, должен существовать еще и некий „универсальный“ механизм, ответственный за изменение критической температуры в тонких пленках сверхпроводников.

Строго говоря, в настоящей работе осцилляции критической температуры в зависимости от толщины пленки $T_{c}(d)$, наблюдение которых было бы сильным ар- 


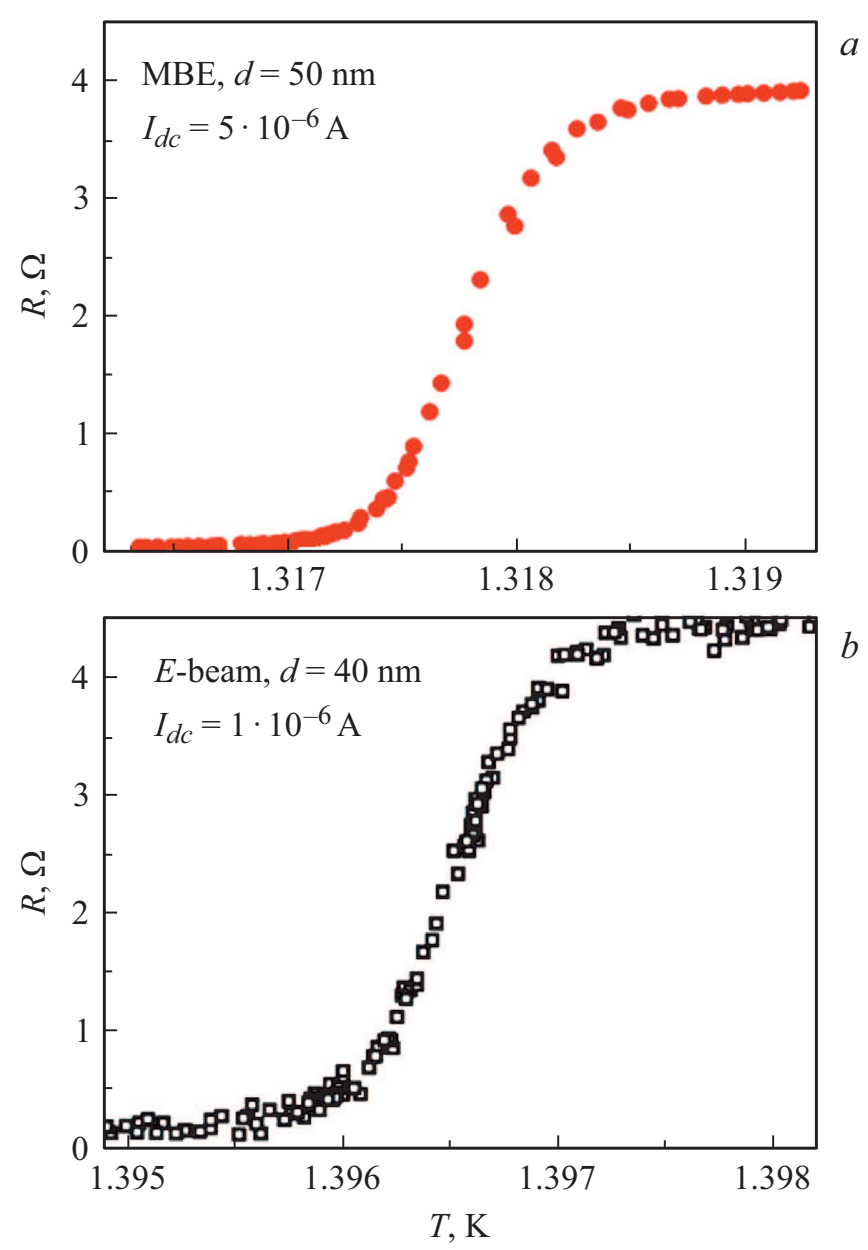

Рис. 3. Зависимость сопротивления от температуры $R(T)$ для алюминиевой пленки: $(a)$ с толщиной $d=50 \mathrm{~nm}$, выращенной методом молекулярно-лучевой эпитаксии; и $(b)$ с толщиной $d=40 \mathrm{~nm}$, полученной испарением электронным лучем.

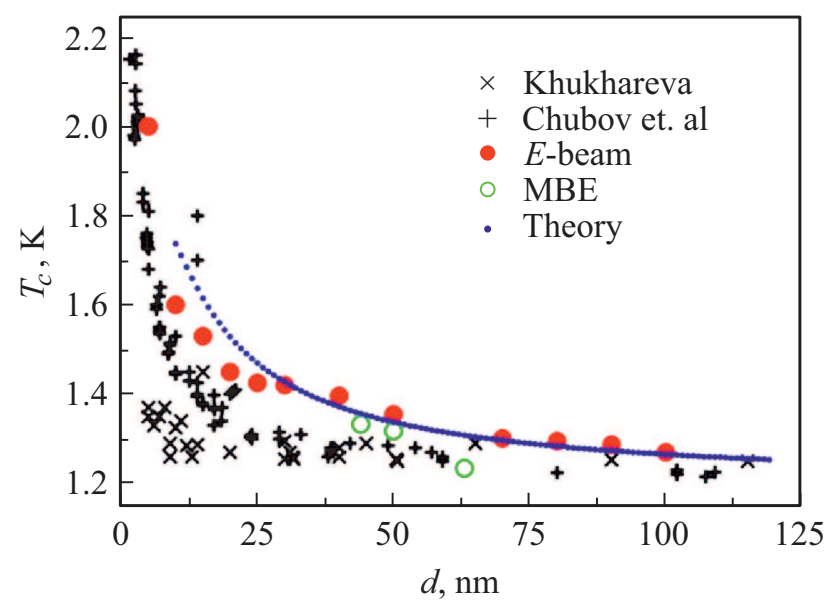

Рис. 4. Зависимость критической температуры $T_{c}$ пленки алюминия от ее толщины $d$. Сплошные кружки $(\bullet)-$ образцы, полученные методом испарения электронным лучем; окружности (о) - образцы, полученные методом молекулярнолучевой эпитаксии; кресты $(\times)$ - на подложках из стекла [3]; кресты (+) - на подложках из стекла и/или кварца [4]; точки $(\cdot)$ - теоретический расчет. Критическая температура массивного алюминия $T_{c}$ (массив) $=1.19 \mathrm{~K}$. гументом в пользу наличия КРЭ, не наблюдались по причине невозможности изготовления нашими методами однородных по толщине пленок с точностью до одного атомного монослоя. Однако, в недавней работе [9] было показано, что учет квантового размерного эффекта, приводящего к перенормировке плотности электронных состояний и константы электрон-фононного взаимодействия, позволяет адекватно описать рост $T_{c}$ в сверхпроводящих пленках алюминия за счет „усреднения“ осциллирующей зависимости $T_{c}(d)$. Исходя из сказанного, нами делается вывод, что представленные экспериментальные результаты могут свидетельствовать в пользу адекватности модели на базе уравнений Боголюбова-де Жена с учетом КРЭ [6-7].

\section{5. Заключение}

Были экспериментально исследованы высококачественные поликристаллические пленки сверхпроводящего алюминия на подложках из GaAs. Был обнаружен заметный рост критической температуры сверхпроводящего перехода с уменьшением толщины пленки. Результаты хорошо коррелируют с более ранними наблюдениями на аналогичных образцах изготовленных различными методами и на различных подложках, что подтверждает универсальность явления. Эффект интерпретируется как проявление квантового размерного эффекта, влияющего как на плотность состояний электронов, так и на электрон-фононное взаимодействие. Теоретический анализ на базе уравнений Боголюбова-де Жена дает удовлетворительное количественное согласие с данными эксперимента. Хочется верить, что наши результаты послужат стимулом для дальнейших исследований.

\section{Благодарности}

Публикация подготовлена в ходе работы совместного Российско-Греческого проекта RFMEFI61717X0001 и T4 $\Delta \mathrm{P} \Omega-00031$. Анализ результатов и подготовка текста статьи проводились КЮА и были поддержаны грантом № 19-01-050 в рамках Программы „Научный фонд Национального исследовательского университета „Высшая школа экономики“ (НИУ ВШЭ)“ в 2019-2020 гг. и в рамках государственной поддержки ведущих университетов Российской Федерации „5-100“.

\section{Конфликт интересов}

Авторы заявляют, что у них нет конфликта интересов.

\section{Список литературы}

[1] A.I. Shal'nikov. Nature 9, 142 (1938).

[2] T.F. Stromberg, C.A. Swenson. Phys. Rev. Lett. 9, 370 (1962).

[3] И.С. Хухарева. ЖЭТФ 43, 1173 (1963). 
[4] П.Н. Чубов, В.В. Еременко, Ю.А. Пилипенко. ЖЭТФ 55, 752 (1968).

[5] В.Л. Гинзбург. ЖЭТФ 47, 2318 (1964).

[6] A.A. Shanenko, M.D. Croitoru,F.M. Peeters. Europhys. Lett. 76 3, 498 (2006).

[7] A.A. Shanenko, M.D. Croitoru, F.M. Peeters. Phys. Rev. B 75, 014519 (2007).

[8] D.A. Luh, T. Miller, J.J. Paggel, T.-C. Chiang. Phy. Rev. Lett. 88, 256802 (2002).

[9] K.Yu. Arutyunov, V.V. Zavialov, E.A. Sedov, I.A. Golokolenov, A.A. Zarudneva, K.V. Shein, I.N. Trun'kin, A.L. Vasiliev, G. Konstantinidis, A. Stavrinidis, G. Stavrinidis, M.D. Croitoru, A.A. Shanenko. Phys. Status Solidi RRL 13, 1800317 (2019).

Редактор К.В. Емцев 